\title{
The illusion of prostate-specific antigen decline in patients with metabolic syndrome and insulin
} resistance

Choi HC, Park JH, Cho BL, Son KY, Yoo YJ, Kwon HT

Department of Family Medicine, Seoul National University Hospital, Department of Family Medicine, Healthcare System Gangnam Centre of Seoul National University Hospital, Seoul, South Korea

BJU Int. 2011; 20. doi: 10.1111/j.1464-410X.2011.10225.x. [Epub ahead of print]

Study Type - Symptom prevalence (prospective cohort) Level of Evidence 1b What's known on the subject? and What does the study add? Studies have shown that PSA is negatively associated with obesity as a result of hemodilution or metabolic effect. Hemodilution could be the main reason for low PSA levels in obese men. However, the intrinsic metabolic effects such as insulin resistance (IR) or metabolic syndrome (MS) on PSA level have not been clearly evaluated although obesity is closely tied to MS and IR. We regarded MS and IR as the pathophysiological cornerstone of metabolic disorder in obesity and analyzed the relationships among MS, IR, and PSA levels, and plasma volume by using the concept of PSA mass, the total circulating PSA protein. PSA mass did not change depending on the severity of the obesity, MS or IR. Even the group with both MS and IR, which could be the most metabolically disturbed in this study, did not have different PSA mass, comparing with the group without any MS or IR. Thus, the decline in PSA level in men with MS or IR can be also explained by increased plasma volume other than any intrinsic metabolic effects.

Objective: To investigate the detailed mechanism of prostate-specific antigen (PSA) decline in metabolic syndrome (MS) and insulin resistance (IR), which lowers the predictive value of the PSA test, we examined the effect of haemodilution and the possibility of an intrinsic metabolic effect.

Patients and Methods: We analysed 28.315 men who underwent routine check-ups. We compared the ageadjusted mean PSA levels in subjects with and without MS before and after adjusting or stratifying the plasma volume. We analysed changes in PSA level, plasma volume and PSA mass according to obesity grade, number of MS components, IR severity and diagnosis of MS, IR or both using an analysis of covariance.

Results: The PSA levels were lower in the group with MS than in the group without MS $(\mathrm{P}=0.001)$, but this difference disappeared after adjusting or stratifying the plasma volume ( $P>0.05$ for all). The PSA levels decreased, plasma volume increased, and PSA mass did not change as the number of MS components increased $(\mathrm{P}=0.002, \mathrm{P}<0.001, \mathrm{P}=0.55$, respectively) or the IR severity increased $(\mathrm{P}=0.001, \mathrm{P}<0.001, \mathrm{P}=0.34$, respectively). Similarly, PSA levels were lower, plasma volumes were higher and PSA masses were the same in subjects with $\mathrm{MS}(\mathrm{P}=0.002, \mathrm{P}<0.001, \mathrm{P}=0.10$, respectively $), \mathrm{IR}(\mathrm{P}=0.018, \mathrm{P}<0.001, \mathrm{P}=0.94$, respectively), or both $(\mathrm{P}=0.003, \mathrm{P}<0.001, \mathrm{P}=0.86$, respectively) than in subjects without those conditions.

Conclusion: The PSA decline in MS and IR may result simply from a haemodilution effect and be unrelated to intrinsic metabolic disturbances. For this reason, PSA levels could be underestimated in patients with MS or IR because of haemodilution.

\section{Editorial Comment}

Prostate-specific antigen (PSA) is the most widely used marker for prostate cancer screening. However, the presence of other benign processes and a myriad of situations may confound the interpretation of PSA levels.

Authors present an elegant cross-sectional study regarding conditions that could affect the validity of PSA tests in prostate cancer screening and showed no difference in PSA levels between subjects with and without metabolic syndrome after adjusting for the plasma volume.

The decrease in PSA levels observed in patients with metabolic syndrome (MS) or insulin resistance (IR) could simply be the result of a haemodilution effect and not related to intrinsic metabolic disturbances. 
Thus, when interpreting PSA levels in men with MS or IR, we should consider that the PSA level could be underestimated because of haemodilution.

Although the utilization of the concept of PSA mass is a virtue, most of study limitations were recognized by authors and further analyses considering direct measurement of plasma volume using an isotope, prostate volume and visceral adipose tissue together are necessary to define the relationships and the use of such calculations for PSA evaluation must also be validated.

On top of that, these patients are commonly exposed to drugs such as nonsteroidal anti-inflammatory (NSAID; $\mathrm{P}=0.03)$, statin $(\mathrm{P}=0.01)$, and thiazide diuretic $(\mathrm{P}=0.025)$ that intake was recently inversely related to PSA levels (1). In this setting, adjusting for potential confounders including demographics, clinical characteristics, physical examination, laboratory studies, and duration of medication use are warranted once five years of NSAID, statin, and thiazide diuretic use was associated with PSA levels lower by $6 \%$, $13 \%$, and $26 \%$, respectively. The combination of statins and thiazide diuretics showed the greatest reduction in PSA levels: $36 \%$ after 5 years. On the other hand, concurrent calcium channel blocker use minimizes or negates the inverse relationship of statin use and PSA level.

\section{Reference}

1. Chang SL, Harshman LC, Presti JC Jr.: Impact of common medications on serum total prostate-specific antigen levels: analysis of the National Health and Nutrition Examination Survey. J Clin Oncol. 2010; 28: 3951-7.

Dr. Leonardo Oliveira Reis Assistant Professor of Urology University of Campinas, Unicamp

Campinas, São Paulo, Brazil

E-mail: reisleo@unicamp.br 